\title{
Use of CRABEL Scores to improve Quality of Medical Records Documentation in Hospitals
}

\section{Arif Raza}

\section{ABSTRACT}

Introduction: This study is based on an approach employed by a medical college hospital for improving the adequacy of documentation in their medical records. The hospital utilized CRABEL scoring tool to screen and score their medical records and then used this information as a feedback to their clinical departments for encouraging them to improve their record documentation.

Aim: The study aims to determine whether the approach of the hospital resulted in any significant change in adequacy of their medical record documentation.

Materials and methods: Baseline sample of 250 current medical records (stratified random) from four clinical departments were scored using CRABEL scoring method to determine baseline average score and number of files with high scores (score $>0.85$ ). Feedbacks on scores were given to departments, along with the information on areas for improvement. Scoring and feedback were repeated every month for six consecutive months, with sample size of 230 to 271 . Trends in average score and number of files with high scores were observed. Difference between average scores of baseline sample and sample at the end of 6 months was statistically tested. Number of files with high scores, in departments where approach was carried out was compared with number of files with high scores, in departments were approach was not carried out, to check statistically significant difference, if any

Results: The trend showed a continuous monthly improvement in both average scores and number of files with high scores. Improvement was found in files of all clinical departments with minor variations. The chi-square test and Student's t test showed a significant difference at $p<0.05$ ( $p$ for chi square 0.001 and for t-test -0.04 ).

Conclusion: The hospital's approach was found to be successful in improving the adequacy of documentation in medical records.

Clinical significance: Medical record constitutes the most important record in a clinical setting. Completeness of medical record is essential for proper patient care, but is a challenge in most organization. The approach has proven successful in this study and can be replicated in other settings for improvement.

Keywords: Case-control study, CRABEL, Documentation of records, Medical record, Scoring of medical record.

\section{Assistant Professor}

Department of Health Care Management, Goa Institute of Management, Panjim, Goa, India

Corresponding Author: Arif Raza, Assistant Professor Department of Health Care Management, Goa Institute of Management, Panjim, Goa, India, Phone: +918322366719 e-mail: arifraza@rediffmail.com
How to cite this article: Raza A. Use of CRABEL Scores to improve Quality of Medical Records Documentation in Hospitals. Int J Res Foundation Hosp Healthc Adm 2016;4(1):5-10.

\section{Source of support: Nil}

Conflict of interest: None

\section{INTRODUCTION}

Medical record is a written accounts of investigations, treatment, and health status of a patient. ${ }^{1}$ Professional documentation by clinical staff is an integral part of practice to ensure safe and effective care. ${ }^{1}$ The purposes served by documentation in medical record are communication, accountability, legislative requirement, quality improvement, and research. ${ }^{1}$ Good record keeping has been mentioned as one of the fundamental requirement for clinical practice in any health care field. ${ }^{2}$ Good quality and real-time documentation in medical record are described as important factors for preventing medical errors. ${ }^{3}$ Health care accreditation bodies, such as Joint Commission International (JCI) and National Accreditation Board for Hospitals and Health care Providers (NABH) have specific standards on medical records documentation which are important for hospitals to comply with for accreditation.

However, concerns over the quality of documentation in medical records in hospitals have been often raised through various means. Inadequacies in medical record documentation have been reported as one of the most common nonconformities in NABH assessments findings. ${ }^{4}$ Gross inadequacies in standards of medical record keeping have been found in several studies and audits. ${ }^{5-7}$ Considerable room for improvement in medical record keeping has also been identified. ${ }^{5}$ Substandard documentations in various categories that include regular update of notes, postoperative instructions, comments about postoperative recovery, the records of advice given to relatives, and incorrect consents have been found. ${ }^{7}$ Need for legibility and use of approved abbreviation in medical records have been stressed upon. ${ }^{8}$ Medical care documentation as an evolving area in the Health care industry of India that needs the attention of experts has also been expressed. ${ }^{8}$ Spillover of problems in primary care with lack of information in records after discharge of patient from hospital has been identified in an audit study. ${ }^{7}$ 
Standard proformas are commonly used in medical record to aid in documentation, but it has been found that alone do not improve the quality of recording. ${ }^{9}$ Another approach is electronic medical records that are being increasingly used and are considered to be a solution to missing entries. However, a large portion of hospitals in India still uses paper-based medical records. Besides, several studies have reported that the quality of recording where physicians are involved did not show improvement with electronic medical record. ${ }^{10-12}$

The CRABEL score as method for scoring the individual medical records on their quality of documentation was described by Crawford et $\mathrm{al}^{13}$ in Annals of Royal College of Surgeons of England. The objective criteria used in the method were derived from the guidelines published by The Royal College of Surgeons.

Crawford et $\mathrm{al}^{13}$ reported that by using CRABEL, scores of medical records typically increased over consecutive audits. As per the authors, CRABEL score is a quick, easy, and reproducible method of assessing and evaluating the quality of medical records. Different studies on CRABEL tool reported improved quality of medical records by using CRABEL as an audit tool. ${ }^{9,14,15}$

Use of CRABEL method has not been reported by any Indian hospital till the time of writing this paper.

\section{BACKGROUND OF THE STUDY}

This study is based on an approach used by a large tertiary care teaching hospital in Gujarat, India, to address the problem of inadequacies and incompleteness of open medical record. The need was felt more as the hospital was trying to get accreditation by $\mathrm{NABH}$ and appropriate medical record keeping was one of their important standard requirements, which hospital was finding tough to meet. Quality team constituted by the hospital for accreditation preparation initiated screening open medical records to comply with this requirement. Initially, screening with randomly prepared checklist revealed numerous discrepancies and incompleteness in records. Based upon these, doctors and nurses were asked to improve their documentation on specific deficiency areas. However, no significant improvement was perceived despite several reminders and follow-ups. Few doctors also raised the issue with the screening checklist stating that it unduly stresses insignificant aspects of medical records. To counter these issues, it was decided to use an accepted standard method of screening and scoring medical record, CRABEL score, and systematically monitor the progress. Since the focus was on improving the real-time documentation quality, the screening and scoring was done only for open medical records.

\section{MATERIALS AND METHODS}

The CRABEL score method was selectively used in four clinical departments (General Medicine [Med.], General Surgery [Sur], Obstetrics \& Gynaecology [O\&G] and Orthopaedics [Ortho]) as they comprised maximum workload of the hospital. The activity was carried out for a period of six months. For each department, medical records selected through stratified random sampling were screened using CRABEL scoring sheet. Each medical record was awarded a score as described by Crawford et. al, The score was related to initial clerking, subsequent entries, consent and discharge letter. From a starting score of 100, one point was deducted for each omissions (deficiency) to arrive at balance final score. However, if one or more points from CRABEL score was not applicable to a particular medical record, the percentage was calculated based on maximum possible score for that file and the score obtained by it. A proforma of CRABEL scoring sheet is given in Annexure 1.

Three members of quality team were trained in-house in the scoring method. The process of screening and scoring was done once per month (generally on middle of the month), for the consecutive 6 months in all the above-mentioned departments.

Only those open medical records were considered for which 24 hours duration has elapsed after patient's admission. This was the minimum time required to ensure that the record has all components of screening required for scoring. Data regarding all relevant admissions was obtained from the computerized hospital information system (HIS) from which records were selected using stratified randomization. The open records were screened to complete the section of 'Initial Clerking', 'Subsequent Entries' and 'Consent'. The section of 'Discharge notes' was completed after patient is discharged. The member screening a particular record keeps a track of his/her records, through HIS, to ensure that section of 'discharge notes' gets completed as soon as patient gets discharged. Once the form is completed for a record, the data was entered in a data sheet created on Microsoft excel, for analysis.

After screening and scoring, the findings were shared with the heads of the departments and with doctors and nurses of the concerned department. The scoring sheet of each medical record of a department was given back to them which shows its score and omissions. Corresponding deficient entries in the record were highlighted for their quick reference. They were asked to improve the score by reducing the number of omissions.

From scores of individual medical records, average score and number of medical records with high score were derived as measures for comparison. Taking reference from other similar studies a benchmark score of 85 $(>84)$ was taken as the cut off to identify medical records with high score. ${ }^{13,16,17}$ 


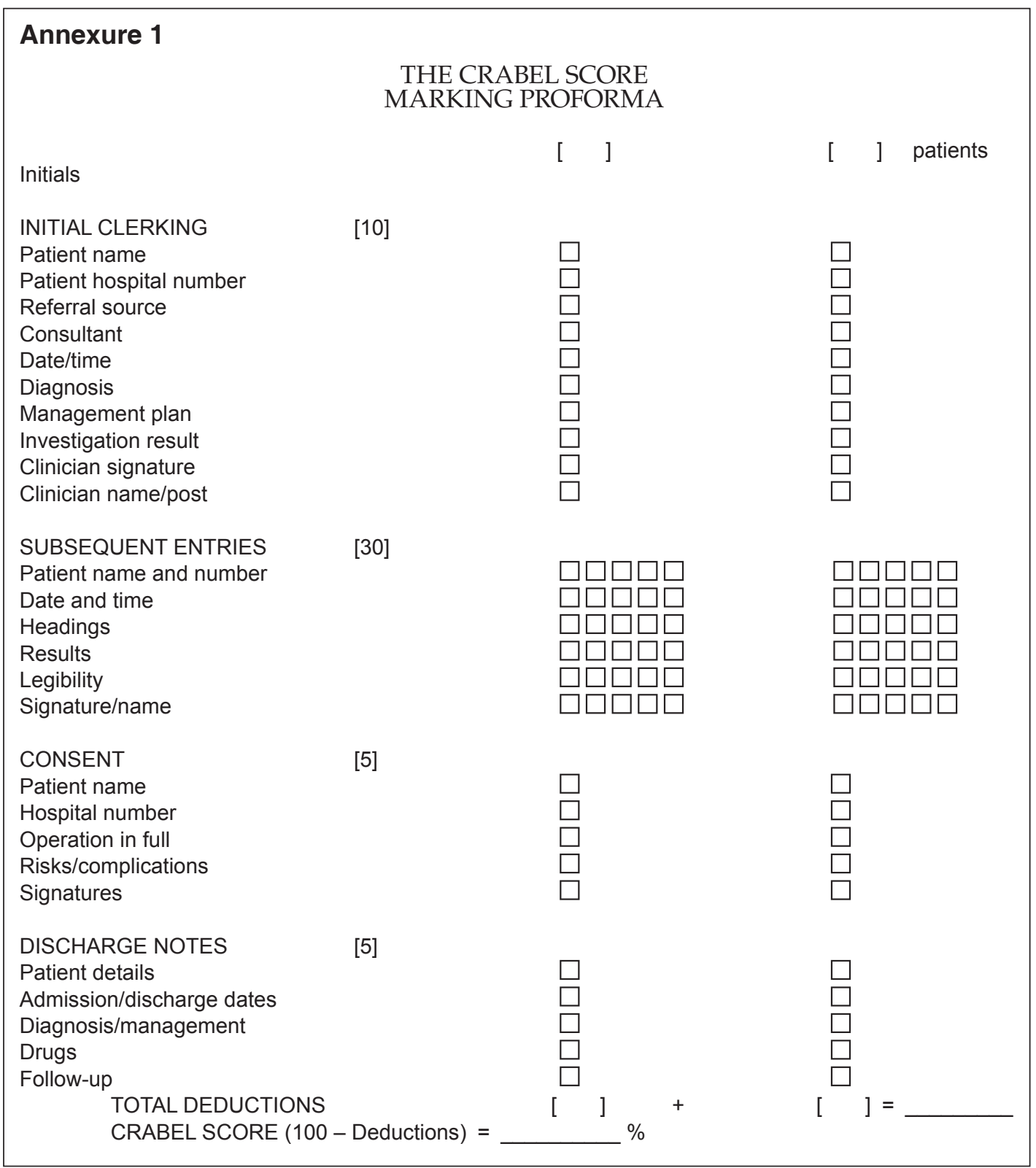

These measures were calculated for each months separately and compared using line graph to observe changes. Based upon the observation a working hypothesis was created to test whether there is a significant change in scores of medical records in 1st month (that is baseline) and last month. The hypothesis was statistically tested using chi-square test and Student t-test.

Finally, the findings from observation and result of statistical testing were discussed in light of other contributory factors to identify limitations of the study and to make a valid conclusion.

\section{DATA AND FINDINGS}

\section{Sampling}

A total of 1,675 open medical records were screened and scored using CRABEL method for 6 months duration. Out of this, scorings of 83 records were incompletely done and scorings of another 19 records had methodical inconsistency. The scores of these 102 records were removed and further calculations were based on remaining 1,573 effective scores. Month-wise and department-wise total medical records screened and the effective number of records for which a valid score were obtained and given in Table 1.

The incompleteness and inconsistencies in scoring method gradually reduced as the members doing the scoring improved their skills with practice. As a result, a gradual increase in proportion of effective to total numbers can be observed in Table 1 .

\section{Measurement and Comparison}

Using excel, average scores, standard deviation, and number of records with high scores were computed. The measures obtained for all records scored in a month are 
Table 1: Total and effective number of open medical records screened and scored

\begin{tabular}{|c|c|c|c|c|c|c|c|c|c|c|}
\hline \multirow{2}{*}{$\begin{array}{l}\text { Dept. } \\
\text { Month }\end{array}$} & \multicolumn{2}{|c|}{ Med } & \multicolumn{2}{|c|}{ Sur } & \multicolumn{2}{|c|}{ O\&G } & \multicolumn{2}{|c|}{ Ortho } & \multicolumn{2}{|c|}{ Total } \\
\hline & $T$ & $E$ & $T$ & $E$ & $T$ & $E$ & $T$ & $E$ & $T$ & $E$ \\
\hline 1 & 83 & 74 & 71 & 64 & 66 & 59 & 62 & 53 & 282 & 250 \\
\hline 2 & 89 & 82 & 74 & 69 & 65 & 61 & 64 & 57 & 292 & 269 \\
\hline 3 & 78 & 73 & 67 & 64 & 62 & 60 & 57 & 52 & 264 & 249 \\
\hline 4 & 83 & 79 & 71 & 68 & 65 & 63 & 60 & 56 & 279 & 266 \\
\hline 5 & 79 & 77 & 70 & 68 & 64 & 61 & 61 & 55 & 274 & 261 \\
\hline 6 & 85 & 83 & 74 & 73 & 64 & 64 & 61 & 58 & 284 & 278 \\
\hline Total & 497 & 468 & 427 & 406 & 386 & 368 & 365 & 331 & 1,675 & 1,573 \\
\hline
\end{tabular}

T: Total number of medical records screened and scored; E: Effective number of records whose scores were considered for further calculations

given in Table 2. As can be observed in the table, the average score has consistently improved every month and achieved a total increase of 11.83 at the end of 6 th month. The number of records with high scores also increased by 13 . As the sample size did not vary much in any of the month, absolute number of records with high score gives a fair idea of comparison and percentage comparison was not required.

Data of individual department's medical records also showed a similar trend in average score and high score records (HSRs), with minor fluctuations.

The department-wise and month-wise trend of change in average scores and HSR is presented in Graphs 1 and 2, which show generally improving trend

Table 2: Measures of comparison for all records scored (month-wise)

\begin{tabular}{lllllll}
\hline Month & $n$ & $\begin{array}{l}\text { Average } \\
\text { score }\end{array}$ & $\begin{array}{l}\text { Std. } \\
\text { dev. }\end{array}$ & $\begin{array}{l}\text { Min. } \\
\text { score }\end{array}$ & $\begin{array}{l}\text { Max. } \\
\text { score }\end{array}$ & HS $^{*}$ \\
\hline 1 & 250 & 57.83 & 9.80 & 40 & 87 & 10 \\
2 & 269 & 58.95 & 9.72 & 39 & 89 & 10 \\
3 & 249 & 63.54 & 9.30 & 46 & 88 & 14 \\
4 & 266 & 67.30 & 8.73 & 50 & 89 & 16 \\
5 & 261 & 67.68 & 8.47 & 47 & 90 & 19 \\
6 & 278 & 69.66 & 8.25 & 48 & 89 & 23 \\
\hline
\end{tabular}

*HS: Number of medical records with high score (i.e., 85 or more)

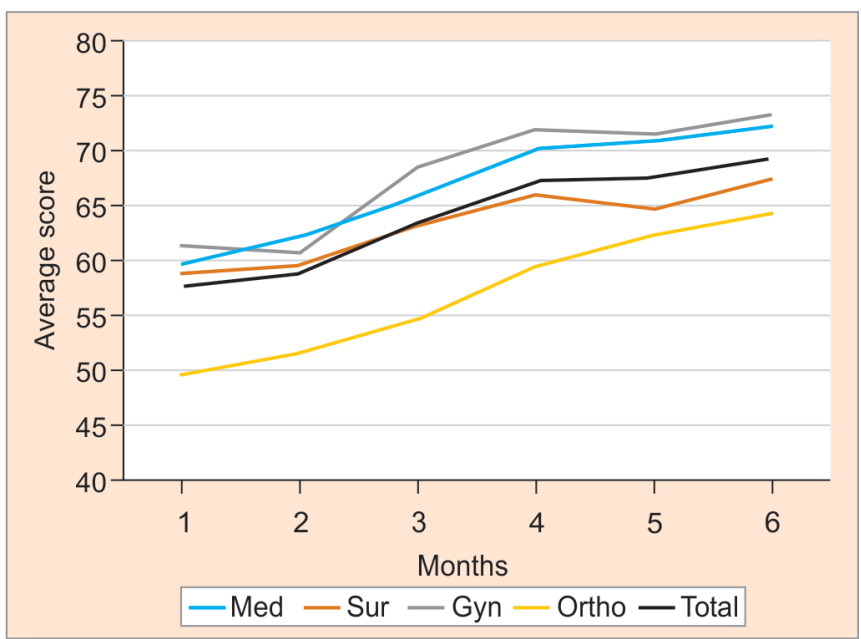

Graph 1: Trend of average CRABEL scores in both average score and number of records with high scores for all departments, with minor fluctuation.

\section{Statistical Testing}

Based on the observations in Table 2 and Graphs 1 and 2, it was hypothesized that CRABEL scoring clubbed with continuous feedback resulted in increase in average score and increase in number of records with high scores in open medical records, in 6 months time.

To test the hypothesis statistically, an independent statistical comparison was made between medical records of departments where the CRABEL scoring was carried out (study group) and in departments where it was not (control group).

A sample of 100 open medical records from corresponding 6th month of the study was selected from control group using proportionate stratified simple random sampling and scored using CRABEL method. Criteria used for sample selection in study department were also used in this controlled sample. The data of scores achieved from this was compared with the data of scores of 278 files from study group (the 6th month scores).

Brief description of the sample of medical records selected and scored from control group is given in Table 3.

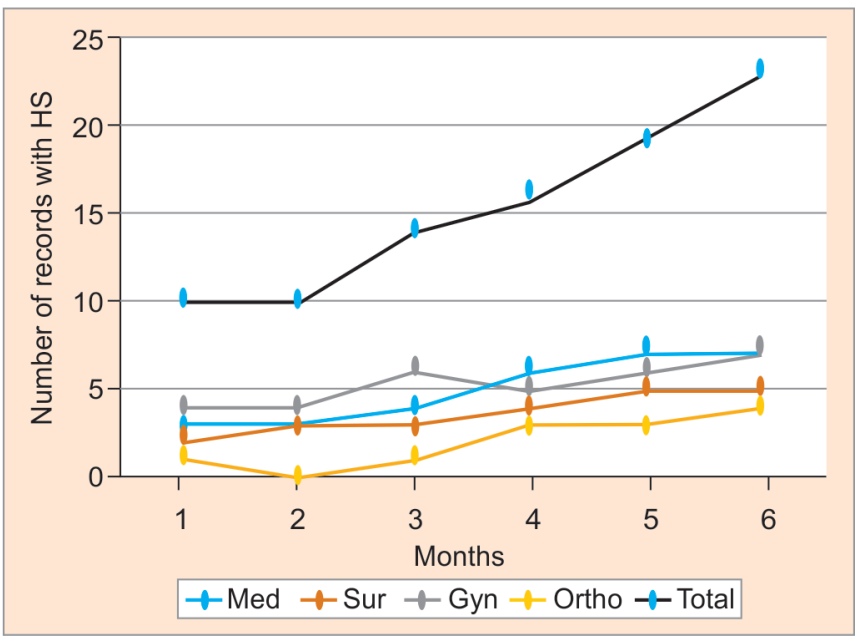

Graph 2: Trend in number of records with high score $(\geq 85)$ 


\begin{tabular}{ll}
\hline \multicolumn{2}{c}{ Table 3: Sample and scoring from control group } \\
\hline Number of records in sample & 100 \\
Distribution & $\begin{array}{l}\text { Pediatrics - 48, ENT - 04, } \\
\text { Ophthalmology - 08, } \\
\end{array}$ \\
& Dermatology - 03, Chest \\
& Medicine - 37 \\
Average score & 61.35 \\
Standard deviation & 11.40 \\
Minimum score & 37 \\
Maximum score & 87 \\
No. of records with HS & 3 \\
\hline
\end{tabular}

Student's t-test (two-tailed, homoscedastic) was performed, using excel statistics function to check if there is any significant difference between average score of control group and study group. The t-value was found to be 7.645 and p-value was less than 0.0001, which showed a highly significant difference between the average scores of control and study group records. The medical records of study group had a better average CRABEL scores as compared to control group.

Chi-square test was performed using excel statistic function to check if there is significant difference in the frequency of occurrence of records with high scores between control and study group. The chi-square value was found to be 4.6862 and p-value at 0.030405 . Thus, at significance level of $p<0.05$, it was found that number of medical records with high CRABEL scores are more frequent in study group records as compared to control group records.

\section{DISCUSSION}

Although, the observation from data and findings from statistical analysis shows a significant improvement in documentation quality of medical records, there are some confounding factors that may have affected the result. Since the focus of the approach was to improve the documentation quality and not primarily to test effectiveness of CRABEL scores, adjusting for confounders were not desired at that time. Some of the factors that may have affected the result are: Increased focus on study group departments, individual differences of doctors and nurses in making entries in records, individual differences in type of disease and care being rendered to different patients, pressure on staff to be ready for accreditation assessment, subjective variation in scoring by different members of quality team, and other quality improvement measures indirectly affecting medical records documentation quality.

Certain other observations can also be made that conforms to the result. Along with an increase in average score, the score at 75 th percentile and score at 25 th percentile also increased over a period of 6 months, which shows that number of records achieving better score has increased while number of records achieving lower score has reduced. The trend of change in percentile score is given in Graph 3.

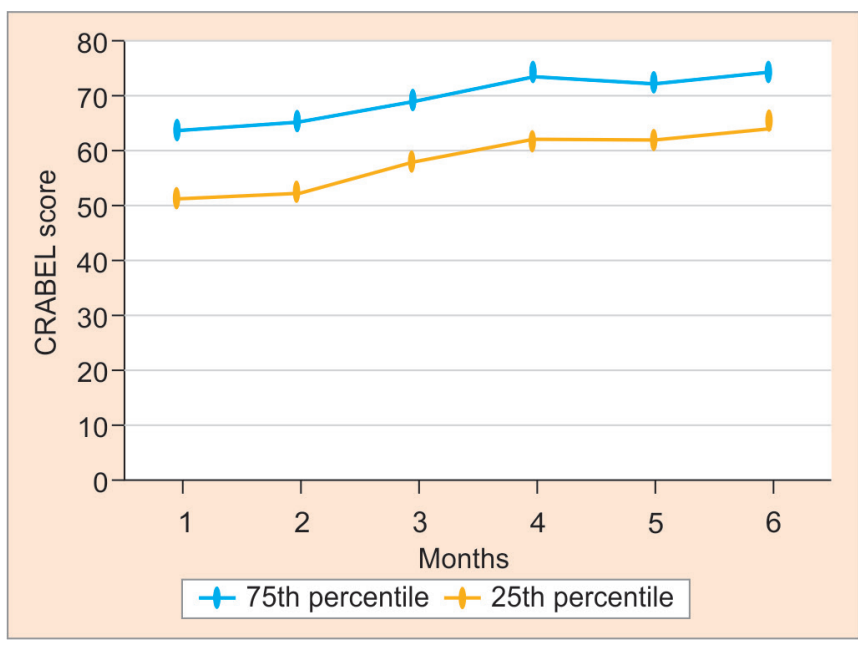

Graph 3: Change in percentile score (75th and 25th) during study period

Standard deviation of the scores also reduced from 9.8 in the 1st month to 8.25 in the last month, indicating better uniformity in documentation across different departments, doctors, and patients.

The trend of changes in scores of medical records from individual departments of the study group were found to be mostly similar to the trend of the total, which indicates the uniformity of result and generalizability to other departments of the hospital.

The study did had certain limitations, which include restriction of the study to one hospital organization, no formal training of team on CRABEL scoring method, and lack of references or benchmarking from similar studies in Indian hospitals.

Based on the above discussion, the conclusion of the study has been carefully presented.

\section{CONCLUSION}

The study showed that the overall approach of CRABEL scoring and feedback was successfully used by the hospital in improving the quality of documentation in medical record. The improvement derived from this approach was statistically significant and hence, can be recommended to be used for the medical records of other departments. However, more studies need to be conducted to rule out the effect of confounding factors. The limitations of the study also need to be overcome for providing evidence of its use on a wider scale and for other hospitals.

\section{CLINICAL SIGNIFICANCE}

Medical record constitutes the most important record in a clinical setting. Completeness of medical record is essential for proper patient care, but is a challenge in most organization. The approach has proven successful in this study and can be replicated in other settings for improvement. 


\section{REFERENCES}

1. WHO. Guidelines for medical record and clinical documentation: WHO-SEARO coding workshop. The World Health Report. Geneva: WHO; 2007 Sep.

2. Kalra D. Medical records. Practitioner 1992 May;236(1514): 533-535.

3. Currie L, Hughes RG. Patient safety and quality: an evidencebased handbook for nurses. Publication No.: 08-043. Rockville (MD): Agency for Health care Research and Quality (US); 2008 Apr.

4. Health care Financial Express. Common deficiencies identified during accreditation assessment surveys [Internet]. Delhi; 2010 [cited 2014 June 2]. Available from: http://Health care.financialexpress.com.

5. Patel AG, Mould T, Webb PJ. Inadequacies of hospital medical records. Ann R Coll Surg Engl 1993 Jan;75(Suppl 1):7-9.

6. Fowkes FG. Audit of the quality of medical records in a district general medicine unit. J R Coll Physicians Lond 1983 Jan 1;17(3):208-212.

7. Twigg J, Briggs T, Parker C, Miller R. Notes: a suitable case for audit. Postgrad Med J 1993 Jul 1;69(813):578-580.

8. Kirubakaran TN. International Health care documentation and common practices. 10th Annual National Conference on "Health Information, Medico Legal Management and Documentation". Pune, India; 2010 Mar.

9. Rai GS, McInnes E, Phongsathorn V, Sharland DE. Medical audit of case notes on a one to one basis. J R Coll Physicians Lond 1991 Oct;25(4):358-359.

10. Pourasghar F, Malekafzali H, Koch S, Fors U. Factors influencing the quality of medical documentation when a paper-based medical records system is replaced with an electronic medical records system: an Iranian case study. International journal of technology assessment in health care. 2008 Oct 1;24(04):445-451.

11. Kern LM, Malhotra S, Barrón Y, Quaresimo J, Dhopeshwarkar R, Pichardo M, Edwards AM, Kaushal R. Accuracy of electronically reported "meaningful use" clinical quality measures: a cross-sectional study. Ann Intern Med 2013 Jan 15;158(2):77-83.

12. David GC, Chand D, Sankaranarayanan B. Error rates in physician dictation: quality assurance and medical record production. Int J Health Care Quality Assur 2014 Mar 3;27(2): 99-110.

13. Crawford JR, Beresford TP, Lafferty KL. The CRABEL score a method for auditing medical records. Ann R Coll Surg Engl $2001 \mathrm{Jan} ; 83(1): 65$.

14. Williams A, Lee P, Kerr A. Scottish Intercollegiate Guidelines Network (SIGN) guidelines on tonsillectomy: a three cycle audit of clinical record keeping and adherence to national guidelines. J Laryngol Otol 2002 Jun 1;116(06):453-454.

15. Lovett BE, Barker R, Ingham C. Spiralling audit sets guidelines and surgical standards: an audit of the completeness of operation notes. Ann R Coll Surg Engl 1998;80(6):44.

16. Jawaid M, Khalique A, Moosa FA, Bakhtiar N. Surgical case notes quality by CRABEL score in Dow University Hospital. J Postgrad Med Inst (Peshawar-Pakistan). 2012 Sep 24;26(4):412-417.

17. Dhariwal DK, Gibbons AJ. The CRABEL score - setting standards in maxillofacial medical note-keeping. Br J Oral Maxillofac Surg 2004 Jun 30;42(3):200-202. 